\title{
A Spatial Signal Processing Review for Practical GNSS Antenna Arrays
}

\author{
Ezequiel A. Marranghelli ${ }^{1}$, G. Ramón López La Valle ${ }^{2}$ and Pedro A. Roncagliolo ${ }^{3}$ \\ UIDET SENyT, Dto. de Electrotecnia, Facultad de Ingeniería, UNLP \\ 48 y 116, Piso 3, La Plata. Argentina \\ 1 emarranghelli@gmail.com \\ ${ }^{2}$ lopezlavalle@gmail.com \\ 3 agustinreing.unlp.edu.ar
}

\begin{abstract}
The performance of an isolated antenna compared with itself but embedded (in-situ) in an array is not the same mostly due to the influence of mutual coupling and electromagnetic diffraction. Although signal processing techniques have been widely spread in literature for antenna arrays of elements with same physical properties and same performance, there is not enough mention regarding antennas with dissimilar behaviors. The usual way of avoiding this problem is by means of some kind of compensation that can reduce the differences but not always eliminate them, unless the information of the active radiation pattern of the antennas in array and wideband compensation techniques are used. A great advantage of employing adaptive methods for processing with antenna arrays is that it ensures good performance even without requiring this complete information. However, the distortions incorporated to the acquired signals by such processing step added to the improper characterization of the antenna array degrades the precision of the received signal parameters measurements. In this paper we analyze the need of using compensation techniques in GNSS applications when the performance of the array of antennas is properly characterized. The degrading effects of using adaptive antenna arrays on acquired GNSS signals and how to avoid them are also evaluated. Simulated results that involve an array of microstrip antennas for GNSS are presented.
\end{abstract}

Resumen- El desempeño de una antena que se encuentra aislada comparada con el de la misma antena embebida (insitu) en un arreglo de antenas es distinto debido mayormente a la influencia del acoplamiento mutuo y la difracción electromagnética. Si bien las técnicas de procesamiento de señales para arreglos de antenas con elementos de misma naturaleza e igual desempeño están ampliamente divulgadas en la literatura, no hay suficiente mención respecto a antenas con comportamientos disímiles. La forma usual de sortear este problema es por medio de una compensación que puede reducir las diferencias, pero no siempre eliminarlas a menos que se emplee la información del diagrama de radiación activo de las antenas dispuestas en arreglo y técnicas de compensación de banda ancha. Una gran ventaja de emplear métodos adaptivos para el procesamiento con arreglos de antenas es que asegura un buen desempeño aún sin requerir esta información completa. Sin embargo, las alteraciones incorporadas a la/s señales adquiridas por dicha etapa de filtrado sumado a la no adecuada caracterización del arreglo de antenas degrada la precisión de las mediciones de los parámetros de las señales recibidas. En este trabajo se analiza la necesidad de emplear técnicas de compensación en aplicaciones de GNSS cuando se tiene adecuadamente caracterizado el desempeño del arreglo de antenas. Se evalúan también los efectos adversos de emplear arreglos de antenas adaptivos sobre las señales GNSS adquiridas y cómo evitarlos. Se presentan resultados simulados que involucran un arreglo de antenas de microtira para GNSS.

\section{INTRODUCTION}

Global Navigation Satellite Systems (GNSS) have become the main choice for obtaining position, velocity and time (PVT) of a vehicle or structure equipped with a receiver [1]. Antenna arrays are currently proposed in many GNSS applications for increasing visibility and improving received signal quality, at the same time that maintain robustness in diverse and changing scenarios.

Undesired perturbations caused by interference, jamming, spoofing signals or even multipath severely degrades the performance of GNSS receivers. A great virtue of employing an antenna array is the possibility of creating a different radiation pattern accordingly with spatial-filtering techniques and therefore it offers an efficient option to mitigate such unwanted signals. Many processing methods have been developed to mitigate these phenomena [2], [3]. However, there exist great difficulties on forcing a radiation pattern with a point or region where null gain is desired to minimize quite enough an electromagnetic interferent wave which impinges on the array and without disrupting the signals of interest [4].

The effects of Mutual Coupling (MC) and Electromagnetic Diffraction (ED) must be taken into account when employing antenna arrays on this kind of applications [5]. These phenomena distort the so called in-situ element radiation patterns in spite of employing identical antennas. The oldest idea to evade differences between array elements behaviour consist of using an array of a very big number of equally distributed antennas to ensure the same relative environment to each element. Nowadays miniaturization is also an essential requirement, therefore small arrays using a reduced number of antennas are highly desired [6], [7]. This work focus on arrays where the so called "large-array assumption" is not applicable.

The radiation pattern of an antenna is a characteristic of great interest because it involves its frequency response with direction of arrival (DOA) dependency. Its modeling is an important topic and should be carefully considered. Classical theory of phased arrays normally supposes a disposition of 
identical antennas with the same behavior. But even in the case of having identical antennas, they will not behave the same being isolated than being embedded in array. For simple array configurations, like thinned dipole antennas, these discrepancies can be mainly explained by MC where the pattern multiplication rule is valid to link active element patterns (insitu patterns) with isolated patterns [8]. For more complex configurations such approximation is less suitable, hence antennas responses may be obtained considering each antenna embedded in array, that means, at its actual electromagnetic environment. In [9] several modeling methods for the radiation patterns of antennas are stated and compared. It is worth to mention that current GNSS receiver technologies allows easily to store in a look-up table enough information about the exact element patterns responses to be directly employed in signal processing stages for small antenna arrays.

Despite several techniques have been previously developed to compensate or minimize the differences between antenna element radiation patterns in order to keep on classical theory of phased arrays [10], [11], frequently variations still exist in greater or lesser extent. This could result in degraded signal processing results as well as non-completely rejected perturbations, particularly when the antenna array has very few elements. These facts impact considerably in interference mitigation and direction finding applications [10]-[12], and specifically in GNSS applications [13]-[17].

As aforementioned, active radiation patterns with strong ED influence could not be explained with a pattern multiplication rule as a linear combination of a common radiation pattern. Therefore, there exist not a particular coupling matrix that leads to such an ideal array of equal antennas. If this approach is used, it can moderate differences or null crossed terms of scattering matrix, but not to exactly compensate the effects except for some particular DOAs of interest [18], [19]. In order to deal with this problem while reducing complexity of receiver and environment characterization many adaptive methods were created, like the ones described in [3]. The central concept of an adaptive antenna array is the use of feedback to optimize some performance index so that it adapts to current signal channels state. They typically ensure robust navigation by rejecting undesired signals while maintaining desired signals availability. However, integrity of desired signals is not always maintained. Typically undesired perturbations are easily mitigated even with minimum knowledge of array performance and impinging signals. But uncertainties of antenna the array performance and underestimation of adaptive antenna impacts can severely distort desired signals if not properly considered by design.

For GNSS receivers the essential information is the time of travel of signals coming from GNSS satellites to the antenna array reference point. This information along with satellites positions allows the PVT computation [1]. GNSS signals that impinges on a receiver must pass through the antenna array, the radio-frequency $(\mathrm{RF})$ front-ends and/or spatial filtering stage. That means signals being modified by the so called Controlled Reception Pattern Antenna (CRPA). Actual generation of robust GNSS receiver designs are focused on determining and avoiding distortions on desired signals' structure caused by these early stages, which otherwise may lead to biases in estimated time of travel. Particularly, these time of travel biases can be mainly approximated with two parameters named phase delay and group delay [20], [21]. Last years research has been focused on improving and reducing complexity of phase and group delay estimations for resilient navigation which implies assuring robustness and accuracy [22], [23]. These phase and group parameters are of great interest for precise positioning systems. Both estimating and/or constraining them with actual techniques require wideband analysis for the CRPA. This is not an easy task and much work is being done for improving these wideband calibration techniques [4], [19], [24], [25]. Total antenna array response is strongly dependent on its geometry, built-in materials, surrounding environment and it is also changeable with time by aging, wheather conditions and also the particular set and structure of impinging signals, perturbations and/or noise.

The paper is organized as follows. In Section II, CRPA scheme and received signals structure along with its parameters of interest are described. In Section III, different techniques for spatial filtering for processing with an antenna array are recalled. In Section IV, the simulated CRPA stages are characterized and results are presented. In Section V, final conclusions are stated.

\section{GNSS RECEIVED SignAl MODEL}

As described in the introduction, spatial filtering techniques can be implemented by deterministic or adaptive methods. Practical receivers implemented with filtering stages are analysed in [4], [23], [26]-[29]. Fig. 1 shows a block diagram of a generic GNSS receiver with an spatial processing (SP) filtering stage. The signals incident on the antenna array from a given direction first propagate through each of the $N$ antenna elements. The array response can be modelled as a time invariant system. Since the individual antenna transfer functions depend upon the direction of each of the incident signals, they will be direction dependent functions. We first define the $\mathbf{g}(f, \theta, \phi)=\left[G_{1}(f, \theta, \phi), \ldots, G_{N}(f, \theta, \phi)\right]^{T}$ vector, with $T$ the transpose operator, that has antenna array far field gain pattern information including direct ray, ED, MC and near field scatterers influences that have been obtained by electromagnetic modeling or calibration measurements. Moreover $\mathbf{s}(f, \theta, \phi)=\left[S_{1}(f, \theta, \phi), \ldots, S_{N}(f, \theta, \phi)\right]^{T}$ is the array manifold which contains the steering vector that is unitary and phase dependent on the geometry of the array [2]. Then the in situ response of the $n^{t h}$ antenna element in a given direction is represented by $S_{n} G_{n}(f, \theta, \phi)$, which represents the total response of an antenna element embedded in array. After reception by the antenna elements, the signal is pre-amplified, passed through a band-limiting filter, downconverted to baseband and digitalized. The front-end and A/D converters stages have the frequency response vector $\mathbf{f}(f)=\left[F_{1}(f), \ldots, F_{N}(f)\right]^{T}$ and $f_{s}$ is the sampling frequency. After down-conversion and digitalization, the signals are fed 


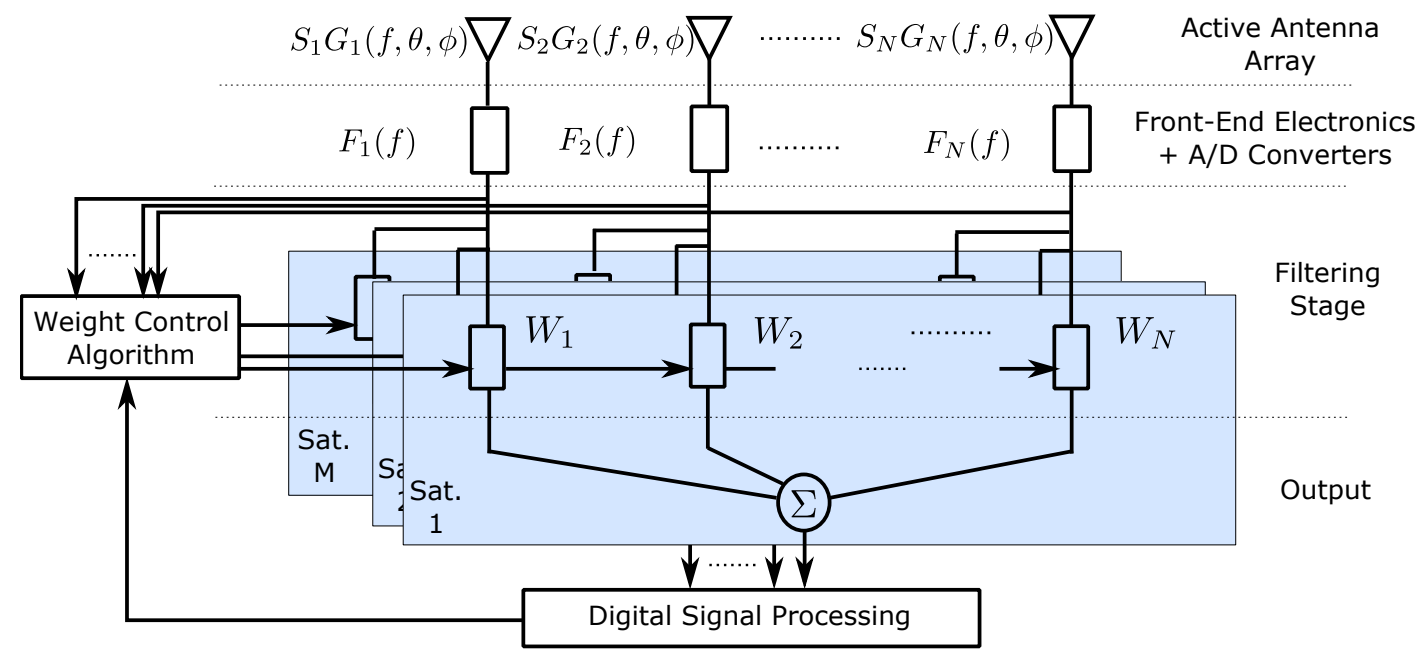

Fig. 1. Model of a GNSS receiver.

into the SP stage to be properly combined together by means of a weight coefficient vector $\mathbf{w}=\left[w_{1}, \ldots, w_{N}\right]^{T}$ to produce the desired output signal. The total baseband frequency response with direction of arrival dependency can be written as

$$
H(f, \theta, \phi)=\mathbf{w}^{H} \mathbf{h}(f, \theta, \phi),
$$

where the superscript $H$ denotes complex transpose or Hermitian operation and

$$
\mathbf{h}(f, \theta, \phi)=\mathbf{s}(f, \theta, \phi) \circ \mathbf{g}(f, \theta, \phi) \circ \mathbf{f}(f) .
$$

In adaptive antenna systems, the weights are typically selected to mitigate interfering signals based on different criteria stated at the Weight Control Algorithm block. It solves and refreshes weight coefficients constantly depending on the received array signals and the information of tracked satellites by the digital signal processing stage. Each desired satellite signal is acquired and tracked if it has enough carrier to noiseinterference ratio (CNIR). High CNIR and minimum phase and group delays distortions is the objective and it must be accomplished by choosing proper weight coefficients. In order to estimate the code and carrier phase biases from the system, we will assume in the following that the SP weights can be read from the processor and are in steady state. Next phase and group delays will be defined.

\section{A. Phase and Group Delays}

Suppose the signal of a GNSS satellite in the frequency domain $d^{F}(f)$ and that there are also $P$ interferent waves $i_{p}^{F}(f)$, with $p=1, \ldots, P$, impinging on the antenna array. The total signal plus interference received by the antenna array, filtered and down-converted by the RF front-end and digitized can be expressed as [22], [23]

$$
\mathbf{x}^{F}(f)=\mathbf{h}\left(f, \theta_{0}, \phi_{0}\right) d^{F}(f)+\sum_{p=1}^{P} \mathbf{h}\left(f, \theta_{p}, \phi_{p}\right) i_{p}^{F}(f),
$$

where subindex 0 indicates the desired signal DOA. Then, a single received signal snapshot vector in the time domain results to be

$$
\mathbf{x}[k]=\int \mathbf{x}^{F}(f) e^{j 2 \pi f k / f_{m}} d f=\left[\begin{array}{c}
x_{1}[k] \\
\ldots \\
x_{N}[k]
\end{array}\right],
$$

where $x_{n}[k]$ denotes the digital output of the $n^{t h}$ front-end channel at an instant $k / f_{s}$.

The received snapshot of signal vector is considered to have three primary components: the desired component $\mathbf{x}_{d}$ that contains the signal of interest, the undesired component $\mathbf{x}_{u}$ which contains the interference, and the thermal noise $\mathbf{n}$ that can be assumed to be independent and identically distributed (i.i.d.). Thus,

$$
\mathbf{x}[k]=\mathbf{x}_{d}+\mathbf{x}_{u}+\mathbf{n} .
$$

Consequently the output of GNSS receiver to the signal processing stage is represented by the sum of the received signal snapshot vector multiplied by the weight vector

$$
y[k]=\mathbf{w}^{H} \mathbf{x}[k] .
$$

Hence, the total signal power $\mathrm{P}$ at the output is

$$
\mathrm{P}=E\left\{y^{*}[k] y[k]\right\}=\mathbf{w}^{H} E\left\{\mathbf{x}[k] \mathbf{x}^{H}[k]\right\} \mathbf{w}=\mathbf{w}^{H} \Phi_{\mathbf{x x}} \mathbf{w},
$$

where $\Phi_{\mathbf{x x}}$ is the total received signal correlation matrix

$$
\Phi_{\mathbf{x} \mathbf{x}}=E\left\{\mathbf{x}^{*}[k] \mathbf{x}^{T}[k]\right\} .
$$

Since the desired and undesired signal components are assumed to be independent, $\Phi_{\mathbf{x x}}$ can also be written as the sum of a desired component, $\Phi_{d}$, undesired component, $\Phi_{u}$, and the i.i.d. thermal noise component, $\sigma^{2} I_{N}$

$$
\Phi_{\mathbf{x x}}=\Phi_{d}+\Phi_{u}+\sigma^{2} I_{N}
$$


with $I_{N}$ the identity matrix of size $N \times N$ and $\sigma^{2}$ the noise variance. The cross-correlation between the antenna array signal vector $\mathbf{x}[k]$ with the locally generated reference signal $d(t+\tau)$ can be expressed as

$$
\phi_{\mathbf{x} d}(\tau)=\mathrm{E}\left\{\mathbf{x}[k] d^{*}\left(k / f_{s}+\tau\right)\right\} .
$$

Then, after beamforming, the reference cross-correlation between the CRPA output and $d(t+\tau)$ yields

$$
\phi_{y d}(\tau)=\mathbf{w}^{H} \phi_{\mathbf{x} d}(\tau) .
$$

Assuming that the GNSS receiver and satellites are perfectly synchronized in time, clock errors can be neglected. Therefore, the time of travel of received signal that is obtained by a non-coherent receiver is determined by finding the peak of the absolute value of (11). The result is influenced by time of travel and also the CRPA biases. However, in [23] a simple least squared approach for phase and group delay biases is defined. Let the phase of total array transfer function be $\alpha\left(f, \theta_{0}, \phi_{0}\right)=\angle H\left(f, \theta_{0}, \phi_{0}\right)$, then it is shown that the antenna-induced carrier phase bias is $\delta \psi_{0} \cong \alpha_{0}$ radians, and the antenna-induced code phase bias is $\delta \tau_{0} \cong-\alpha_{1} / 2 \pi$ seconds, where

$$
\left[\begin{array}{l}
\alpha_{0} \\
\alpha_{1}
\end{array}\right]=\left(A^{T} B A\right)^{-1} A^{T} B \boldsymbol{\alpha} .
$$

In (12), $\mathrm{A}$ is an $M \times 2$ matrix given by

$$
A^{T}=\left[\begin{array}{cccc}
1 & 1 & \ldots & 1 \\
f_{1} & f_{2} & \ldots & f_{Q}
\end{array}\right]
$$

$B$ is a $M \times M$ matrix given by

$$
\begin{gathered}
\operatorname{diag}\left\{\left[\Phi_{d d}\left(f_{1}\right)\left|H\left(f_{1}, \theta_{0}, \phi_{0}\right)\right|, \Phi_{d d}\left(f_{2}\right)\left|H\left(f_{2}, \theta_{0}, \phi_{0}\right)\right|,\right.\right. \\
\left.\left.\ldots \Phi_{d d}\left(f_{Q}\right)\left|H\left(f_{Q}, \theta_{0}, \phi_{0}\right)\right|\right]\right\}
\end{gathered}
$$

and $\boldsymbol{\alpha}$ is a vector of length $M$ given by

$$
\boldsymbol{\alpha}^{T}=\left[\alpha\left(f_{1}, \theta_{0}, \phi_{0}\right), \alpha\left(f_{2}, \theta_{0}, \phi_{0}\right), \ldots, \alpha\left(f_{Q}, \theta_{0}, \phi_{0}\right)\right] .
$$

In the above equations, $f_{1}, f_{2}, \ldots, f_{Q}$ are $Q$ frequencies covering the frequency band of the desired signal.

With an ideally behaved antenna array of isotropic elements then the resulting estimated time delay and carrier phase will ideally be $\hat{\tau_{0}}=\tau_{0}$ and $\hat{\psi}_{0}=\psi_{0}$, which corresponds to current delay time and carrier phase of satellite signal when arrives from the transmit satellite antenna reference point to the antenna array reference point at receiver. But for an actual antenna array (11) includes also the CRPA effects. This will lead to the already mentioned undesired biases of phase, $\delta \psi_{0}$, and group delay, $\delta \tau_{0}$, so that delay and phase estimations will be equal to $\hat{\tau}_{0}=\tau_{0}+\delta \tau_{0}$ and $\hat{\psi}_{0}=\psi_{0}+\delta \psi_{0}$. In terms of navigation solutions, group delay biases can produce errors in the order of $c \cdot \delta \tau_{0}$ meters, being $c=3 \cdot 10^{8} \mathrm{~m} / \mathrm{s}$ the speed of light in vacuum, while carrier phase delay is used for a more precise estimation and can produce error in the order of centimeter level. Such biases will be accounted for at section IV.

Since functions (9), (10) and (11) are theoretical values, these correlation functions require to be estimated for practical situations. Hence, for next sections they will be replaced by their estimates, that is

$$
R_{\mathbf{x x}}=\frac{1}{K} \sum_{k=1}^{K} \mathbf{x}[k] \mathbf{x}^{H}[k],
$$

called sampled auto-correlation function and

$$
\begin{gathered}
\hat{\mathbf{r}}_{\mathbf{x} d}(\tau)=\frac{1}{K} \sum_{k=1}^{K} \mathbf{x}[k] d^{*}\left(k / f_{s}+\tau\right), \\
\hat{\mathbf{r}}_{y d}(\tau)=\mathbf{w}^{H} \hat{\mathbf{r}}_{\mathbf{x} d}(\tau),
\end{gathered}
$$

that are the sampled cross-correlation functions. Superindex $*$ denotes complex conjugate.

Next we will consider some well known techniques for SP and commonly employed for GNSS array processing.

\section{SP FILTERING METHODS}

Spatial processing methods are employed for the purposes of increasing the CNIR and to obtain the most clean near to original replica of the desired GNSS signal that arrives to the receiver. Each method meets a different objective and consequently produce different results. Some well known cases will be listed below and will be used for simulations in next section.

\section{A. Phased Array Beamforming (PA)}

In [2] a simplified version of deterministic beamforming, weights $\mathbf{w}_{P A}$ are calculated with a given knowledge of antenna baselines, platform orientation, and incoming signal and interference DOAs. Actually, only the steering vectors $\mathbf{s}(f, \theta, \phi)$ of each DOA of interest and array geometry orientation is needed. With this SP filtering method the number of restrictions can be up to $P<N$, so that $\mathbf{w}_{P A}$ must satisfy

$$
\mathbf{w}_{P A}^{H} \mathbf{s}_{0}=1 \quad \& \quad \mathbf{w}_{P A}^{H} \mathbf{s}_{p}=0,
$$

where $\mathbf{s}_{p}=\mathbf{s}\left(f_{p}, \theta_{p}, \phi_{p}\right) . f_{p}, p=0, \ldots, P$, are the central frequencies of considered signals.

\section{B. Deterministic Beamforming (DB)}

For the deterministic antenna array beamforming, weights $\mathbf{w}_{D B}$ are calculated with a given knowledge of antenna baselines, platform orientation, incoming signal and interference frequency responses, and DOAs and total antenna array gain/phase response [18]. In here, the CRPA is restricted so that for each DOA of interest $\left(\theta_{p}, \phi_{p}\right)$ at a particular frequency $f_{p}$ meets desired requirements for $p=0, \ldots, P$. With this SP filtering method the number of restrictions can be up to $P<N$, so that $\mathbf{w}_{D B}$ must satisfy

$$
\mathbf{w}_{D B}^{H} \mathbf{h}_{0}=1 \quad \& \quad \mathbf{w}_{D B}^{H} \mathbf{h}_{p}=0,
$$

where $\mathbf{h}_{p}=\mathbf{h}\left(f_{p}, \theta_{p}, \phi_{p}\right)$ and $f_{p}, p=0, \ldots, P$ are the central frequencies of considered signals. 


\section{Sectorial Compensation and Beamforming (SC)}

In [10] a pre-processing technique is defined by using a linear transformation matrix that transforms a sequence of measured vectors $\mathbf{h}_{i}=\mathbf{h}\left(f_{i}, \theta_{i}, \phi_{i}\right)$ induced over an embedded antenna array to the amplitude vectors $\mathbf{h}_{\text {ideal }, i}=\mathbf{s}\left(f_{i}, \theta_{i}, \phi_{i}\right)$ that will be produced on an ideal antenna array of identical antennas in a least squares sense. This method requires to define sectors among the field of view. Quantity and size of sectors can be predefined by user. All vectors at the calibration points are combined to form the matrix $V=\left[\mathbf{h}_{1}, \mathbf{h}_{2}, \ldots, \mathbf{h}_{M}\right]$, where $M$ is the number of calibration points. Based on the knowledge of the individual DOAs of the calibration points, the matrix of ideal voltages is formed as $V_{\text {ideal }}=$ $\left[\mathbf{h}_{\text {ideal }, 1}, \mathbf{h}_{\text {ideal }, 2}, \ldots, \mathbf{h}_{\text {ideal }, M}\right]$. For a practical situation with a particular finite number of different calibration points, they are related each other by the following equation

$$
V \approx C V_{\text {ideal }}
$$

Therefore, the coupling matrix is estimated by the least square solution given by

$$
C=V V_{\text {ideal }}^{H}\left(V_{\text {ideal }} V_{\text {ideal }}^{H}\right)^{-1} .
$$

A necessary condition for obtaining a unique solution is that the number of different calibration points must be at least equal to the number of array elements. For our particular case at simulation instance, the calibration points are all selected in the range of $\left(\theta_{p}-5^{\circ} \leq \theta_{i} \leq \theta_{p}+5^{\circ} ; \phi_{p}-5^{\circ} \leq \phi_{i} \leq \phi_{p}+5^{\circ}\right)$, for each $i=1, \ldots, M$, and for all arriving signals $p=0, \ldots, P$.

After compensation, beamforming is performed with previously described PA beamforming technique which, in combination, results in $\mathbf{w}_{S C}$ weight vector given by

$$
\mathbf{w}_{S C}=C^{-1} \mathbf{w}_{P A} .
$$

\section{Power Inversion + Eigenbeamformer $(P I)$}

As described in [28] Power Inversion is an adaptive method that requires the knowledge and inversion of the correlation matrix $\hat{R}_{\mathbf{x x}}$ to remove every strong perturbation. It supposes that GNSS signals will not be much distorted because they are deeply buried into noise, which is a good assumption for GNSS since it employs Direct-Sequence Spread-Spectrum codification [1]. After Power Invertion processing signal vector yields

$$
\hat{\tilde{\mathbf{x}}}=\hat{R}_{\mathbf{x x}}^{-1} \mathbf{x} .
$$

The signal vector $\tilde{\mathbf{x}}$ is free of interferences, so a postcorrelation can be performed to detect a GNSS satellite signal and a blind beamforming can be applied. This is particularly interesting since a peak in the cross correlation function of (11) could not be found in a harmful situation of strong jammers and therefore signal could not be recovered when no temporal or spatial information is available. But evaluating

$$
\hat{\mathbf{r}}_{\tilde{\mathbf{x}} d}(\tau)=\frac{1}{K} \sum_{k=1}^{K} \tilde{\mathbf{x}}[k] d^{*}\left(k / f_{s}+\tau\right),
$$

and then finding the spatial post-correlation matrix $\hat{R}_{\hat{\mathbf{x}}_{\tilde{x} d} \hat{\mathbf{r}}_{\tilde{\mathbf{x}} d}}$, an eigendecomposition of this array correlation matrix is performed so that the array weight vector can be set to the eigenvector corresponding to the largest eigenvalue. This is called Eigenbeamforming and yields to

$$
\mathbf{w}_{P I}=\mathcal{P}\left\{\hat{R}_{\hat{\mathbf{r}}_{\tilde{\mathbf{x}} d} \hat{\mathbf{r}}_{\tilde{\mathbf{x}}} d}\right\},
$$

where $\mathcal{P}$ represents the principal eigenvector operator.

\section{E. Minimum Variance Distortionless Response (MVDR)}

The Minimum Variance Distortionless Response beamformer is a well known adaptive technique for interference mitigation [2]. This algorithm constrains to unity the array gain in a particular look direction and frequency while rejecting coherent interference down to the noise floor. Then, it only considers the DOA of the signal of interest, and minimizes the power of all other impinging signals that arrives from another directions, maximizing the CNIR. It is defined as follows

$$
\mathbf{w}_{M V D R}=\arg \min _{\mathbf{w}}\left\{\mathbf{w}^{H} \hat{R}_{\mathbf{x x}} \mathbf{w}\right\} \quad \text { s.t. } \quad \mathbf{h}_{0}^{H} \mathbf{w}=1,
$$

and its closed form solution is

$$
\mathbf{w}_{M V D R}=\hat{R}_{\mathbf{x x}}^{-1} \mathbf{h}_{0} /\left(\mathbf{h}_{0}^{H} \hat{R}_{\mathbf{x x}}^{-1} \mathbf{h}_{0}\right),
$$

where $\mathbf{h}_{0}=\mathbf{h}\left(f_{0}, \theta_{0}, \phi_{0}\right)$.

\section{F. Least Mean Squares (LMS)}

The Least Mean Square beamformer is another traditional adaptive technique for interference mitigation [2]. It is based in a mean square error minimization to match a desired reference signal to the array output, while rejecting coherent interference present at the array input. It needs a temporal reference, which implies knowing a first estimate of delay time $\hat{\tau}_{0}$ between received and reference signals. Hence the $\mathbf{w}_{L M S}$ weight vector yields

$$
\mathbf{w}_{L M S}=\arg \min _{\mathbf{w}}\left\{\left|\mathbf{w}^{H} \mathbf{x}-d\left(t+\hat{\tau_{0}}\right)\right|^{2}\right\},
$$

and its closed form solution is

$$
\mathbf{w}_{L M S}=\hat{R}_{\mathbf{x x}}^{-1} \mathbf{r}_{0}
$$

where $\mathbf{r}_{0}=\hat{\mathbf{r}}_{\mathbf{x} d}\left(\hat{\tau}_{0}\right)$.

\section{Simulations}

\section{A. Array Design}

Normally, GNSS receivers employ microstrip antennas since they are characterized by having good hemispherical radiation properties, which is important to maximize the visibility range of available satellites. In addition microstrip antennas can be circular polarized, they are low cost, mechanically robust and low profile. In particular, in this work a microstrip antenna array with square patch and truncated corners design was selected to achieve right hand circular polarization (RHCP) [30]. Such antennas have simple coaxial probes and are implemented on a RT/duroid 6002 substrate with relative dielectric 
permitivity $\varepsilon_{r}=2.94$ [31]. It is important to mention that the proposed antenna dimensions were selected for the L1 band of GPS and GLONASS, which increases versatility and functionality. However, at this work the CRPA response will be only analyzed for the GPS L1 bandwidth. The antenna array and its parameters of interest are shown in Fig. 2.

For the array structure, a disposition of four aligned antennas separated by a distance of $d=0.56 \lambda_{c}$ is proposed, being $\lambda_{c}$ the wavelength at central frequency of the L1 band of GPS, $f_{c}=1.57542 \mathrm{GHz}$. The selected distance between array elements is due to a physical restriction imposed by their own dimensions. Ideally a separation of $\lambda_{c} / 2$ would be chosen in order to avoid or minimize possible ambiguities. Since antennas are as closer as possible, MC and ED effects are thus considerable, which justifies this work. Also, each antenna is built with proper substrate and ground plane to reduce propagation of superficial currents among them.

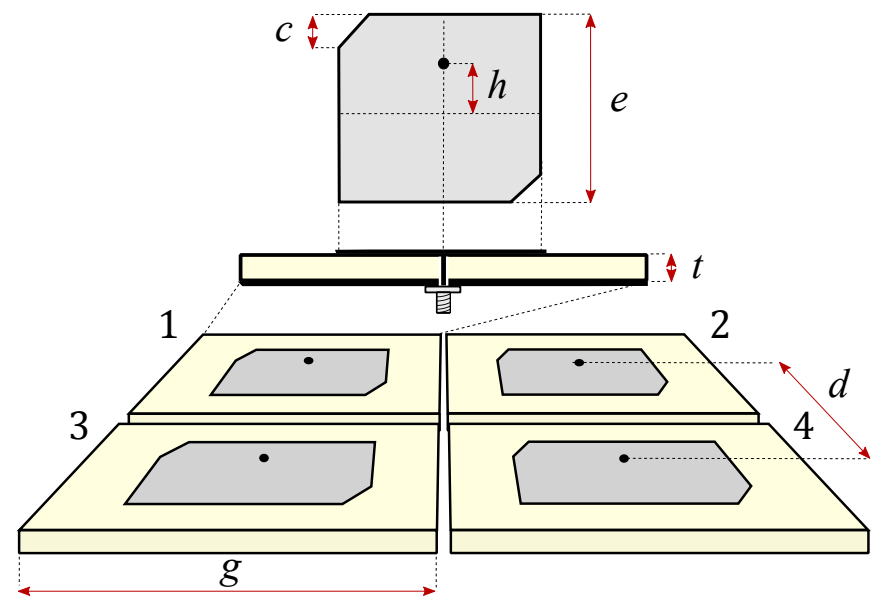

Fig. 2. Patch antenna element and $2 \times 2$ array.

Final dimensions of the already described microstrip antenna array were obtained by simulation with a Finite Integration Method program [32] and are presented in Table I.

TABLE I

AnTENNA Dimensions.

\begin{tabular}{|c|c|}
\hline Parameter & Dimension $[\mathrm{mm}]$ \\
\hline \hline$c$ & 5.2 \\
\hline$h$ & 10.35 \\
\hline$g$ & 100 \\
\hline$e$ & 53.7 \\
\hline$t$ & 1.524 \\
\hline
\end{tabular}

\section{B. RF Front-End and $A / D$ Converters}

A simplified model was selected for the $\mathbf{f}(f)$ transfer function. The assumed receiver employs an RF front-end stage with an homodyne scheme where amplifiers, mixers and filters are ideal. Relevant properties of this stage are:

- Bandwidth: $B_{W}=10 \mathrm{MHz}$

- Cascaded noise figure: $F=0 \mathrm{~dB}$
- Cascaded gain: $G=0 \mathrm{~dB}$

- Sampling frequency: $f_{s}=24 \mathrm{MHz}$

\section{Phase and Group Delay Estimations}

GNSS satellite constellation is distributed so that for a receiver at any point on the Earth's surface exist a minimum number of available satellites at any time. At least four satellites are needed to obtain the navigation solution. Normally, for a receiver with a microstrip antenna the satellite elevations should be in the range of $0^{\circ} \leq \theta \lesssim 70^{\circ}$ measured from a local coordinate system with center at the reference point of the antenna array and with $z$-axis normal to the imaginary plane where it is located. All desired satellites signals can be acquired at the same time with parallel processing stages as shown in Fig. 1.

Simulation examples will be performed with only one satellite signal coming from $\left(\theta_{0}, \phi_{0}\right)=\left(40^{\circ}, 45^{\circ}\right)$ DOA. This DOA is selected as a generic case. At this particular DOA, the frequency response of the CPRA is near to the average of the antenna array radiation pattern at bandwidth of interest.

We are interested in comparing each of the previously described SP methods performance in terms of average (mean) and standard deviation (std) of the estimations of $\delta \tau_{0}$ and $\delta \psi_{0}$, and average values of CNIR and interference-to-signal ratio (ISR) at output. CNIR has units of [dB-Hz] and ISR units of $[\mathrm{dB}]$. For the next proposed scenarios, adopted configuration is

- Noise power density at input: $N_{0}=-174 \mathrm{dBm}-\mathrm{Hz}$

- Carrier to noise density at input: $C / N_{0}=40 \mathrm{~dB}-\mathrm{Hz}$

- Signal DOA: $\left(\theta_{0}, \phi_{0}\right)=\left(40^{\circ}, 45^{\circ}\right)$

- Central frequency: $f_{c}=1.57542 \mathrm{GHz}$

- Integration time of acquired signal: $1 \mathrm{~ms}$

- Number of averaged events: 100

and the assumed jamming attack or interference settings are:

- Signal spectrum: Continuous tone at $f_{c}=1.575 \mathrm{MHz}$

- Number of undesired sources: 3

- Each jamming ISR at input: $50 \mathrm{~dB}$

- DOA: Three random and uniformly distributed locations with $60^{\circ} \leq \theta_{p} \leq 90^{\circ}$ and $0^{\circ} \leq \phi_{p} \leq 360^{\circ}$ for each $p=1, \ldots, P$

- Number of averaged events: Performed with a Monte Carlo simulation over 100 cases

1) Isotropic Antennas, No Jamming: First, an antenna array model with ideal and equally behaved isotropic antennas will be employed. That means antenna gains $G_{n}(f, \theta, \phi)=1$ for every frequency and DOA. Only signal of interest arrives to the receiver array and no jamming/interference is present. Results are listed in Table II.

It is verified that in case of having a distortionless antenna array transfer function at the DOA and bandwidth of interest, there are no delay biases on signal of interest. Besides, with no undesired sources available all methods behave similar in terms of resulting signal quality at output. 
TABLE II

SiMULATION $\mathrm{N}^{\circ} 1$. ISOTROPIC ANTENNAS, NO JAMMING.

\begin{tabular}{|c|cc|r|r|c|c|}
\hline \multirow{2}{*}{$\begin{array}{c}\text { SP } \\
\text { Method }\end{array}$} & \multicolumn{5}{|c|}{ Parameter estimation via Monte Carlo simulation } \\
\cline { 2 - 7 } & \multicolumn{2}{|c|}{$c \cdot \delta \tau_{0}$} & \multicolumn{2}{|c|}{$\delta \psi_{0}$} & \multicolumn{1}{c|}{ CNIR } & \multicolumn{1}{c|}{ ISR } \\
\cline { 2 - 7 } & mean & std & mean & std & mean & mean \\
\hline PA & $0.00 \mathrm{~m}$ & $0.00 \mathrm{~m}$ & $0.0^{\circ}$ & $0.0^{\circ}$ & 49.45 & - \\
DB & $0.00 \mathrm{~m}$ & $0.00 \mathrm{~m}$ & $0.0^{\circ}$ & $0.0^{\circ}$ & 49.45 & - \\
SC & $0.00 \mathrm{~m}$ & $0.00 \mathrm{~m}$ & $0.0^{\circ}$ & $0.0^{\circ}$ & 49.45 & - \\
PI & $0.00 \mathrm{~m}$ & $0.00 \mathrm{~m}$ & $-30.6^{\circ}$ & $144.0^{\circ}$ & 49.45 & - \\
MVDR & $0.00 \mathrm{~m}$ & $0.00 \mathrm{~m}$ & $0.0^{\circ}$ & $0.0^{\circ}$ & 49.45 & - \\
LMS & $0.00 \mathrm{~m}$ & $0.00 \mathrm{~m}$ & $0.0^{\circ}$ & $0.0^{\circ}$ & 49.45 & - \\
\hline
\end{tabular}

2) Isotropic Antennas, Three Narrowband Jammers: The same ideal antenna array model $G_{n}(f, \theta, \phi)=1$ for every frequency and DOA. Now three jamming/interferent sources are present. A Monte Carlo simulation is performed to average results when jammers positions are randomized near the horizon. Results are listed in Table III.

TABLE III

SIMULATION Nº 2 . ISOTROPIC ANTENNAS, 3 JAMMERS

\begin{tabular}{|c|cc|c|c|c|c|}
\hline \multirow{2}{*}{$\begin{array}{c}\text { SP } \\
\text { Method }\end{array}$} & \multicolumn{5}{|c|}{ Parameter estimation via Monte Carlo simulation } \\
\cline { 2 - 7 } & \multicolumn{2}{|c|}{$c \cdot \delta \tau_{0}$} & \multicolumn{2}{|c|}{$\delta \psi_{0}$} & \multicolumn{1}{c|}{ CNIR } & \multicolumn{1}{c|}{ ISR } \\
\cline { 2 - 7 } & mean & std & mean & std & mean & mean \\
\hline PA & $0.00 \mathrm{~m}$ & $0.00 \mathrm{~m}$ & $0.0^{\circ}$ & $0.0^{\circ}$ & 41.75 & -82.76 \\
DB & $0.00 \mathrm{~m}$ & $0.00 \mathrm{~m}$ & $0.0^{\circ}$ & $0.0^{\circ}$ & 41.75 & -82.76 \\
SC & $0.00 \mathrm{~m}$ & $0.00 \mathrm{~m}$ & $0.0^{\circ}$ & $0.0^{\circ}$ & 41.75 & -82.76 \\
PI & $0.00 \mathrm{~m}$ & $0.02 \mathrm{~m}$ & $7.7^{\circ}$ & $148.0^{\circ}$ & 45.79 & -18.56 \\
MVDR & $0.00 \mathrm{~m}$ & $0.00 \mathrm{~m}$ & $0.0^{\circ}$ & $0.0^{\circ}$ & 48.03 & -19.14 \\
LMS & $0.00 \mathrm{~m}$ & $0.00 \mathrm{~m}$ & $0.0^{\circ}$ & $0.0^{\circ}$ & 48.03 & -19.14 \\
\hline
\end{tabular}

It can be seen that when employing different SP methods for jamming/interference mitigation but having a distortionless antenna array transfer function, then biases on signal of interest are still negligible. Then, if an array of isotropic antennas could be performed, then CRPA biases would not be a severe problem.

3) Non-Isotropic Antennas, No Jamming: In this case, the antenna array model of section IV.A is employed and no interferences are considered. Results are listed in Table IV.

TABLE IV

SIMULATION N ${ }^{\circ}$ 3. NON-ISOTROPIC ANTENNAS, NO JAMMING.

\begin{tabular}{|c|cc|r|r|c|c|}
\hline \multirow{2}{*}{$\begin{array}{c}\text { SP } \\
\text { Method }\end{array}$} & \multicolumn{5}{|c|}{ Parameter estimation via Monte Carlo simulation } \\
\cline { 2 - 7 } & \multicolumn{2}{|c|}{$c \cdot \delta \tau_{0}$} & \multicolumn{2}{|c|}{$\delta \psi_{0}$} & \multicolumn{1}{c|}{ CNIR } & ISR \\
\cline { 2 - 7 } & mean & std & mean & std & mean & mean \\
\hline PA & $3.02 \mathrm{~m}$ & $0.00 \mathrm{~m}$ & $97.7^{\circ}$ & $0.0^{\circ}$ & 52.52 & - \\
DB & $3.05 \mathrm{~m}$ & $0.00 \mathrm{~m}$ & $0.0^{\circ}$ & $0.0^{\circ}$ & 52.63 & - \\
SC & $3.04 \mathrm{~m}$ & $0.00 \mathrm{~m}$ & $-0.4^{\circ}$ & $0.0^{\circ}$ & 52.59 & - \\
PI & $3.04 \mathrm{~m}$ & $0.05 \mathrm{~m}$ & $-87.0^{\circ}$ & $10.8^{\circ}$ & 52.13 & - \\
MVDR & $3.03 \mathrm{~m}$ & $0.01 \mathrm{~m}$ & $-0.0^{\circ}$ & $0.1^{\circ}$ & 52.36 & - \\
LMS & $3.05 \mathrm{~m}$ & $0.01 \mathrm{~m}$ & $0.1^{\circ}$ & $0.0^{\circ}$ & 52.63 & - \\
\hline
\end{tabular}

Now, mean values of group and phase delays are severely degraded. And clearly this is not caused by selected SP method but due to the antenna array response, which distorts signal of interest. Moreover, standard deviation maintains reduced because CRPA at a particular DOA is supposed to be stationary in time with the only exception of noise influence on weight coefficients of adaptive methods. However, the biases also change considerably if the DOA of signal of interest is changed as verified in other works [21].

4) Non-Isotropic Antennas, Three Narrowband Jammers: Taking the previous simulation configuration as starting point, three jammers are added and a Monte Carlo simulation performed. This case allows to particularly demonstrate secondary effects of deterministic and adaptive SP methods over received signal and the ability of rejection of undesired sources when non-isotropic antennas are used and jamming blockage must be avoided. Results are listed in Table V.

TABLE V

Simulation $\mathrm{N}^{\circ} 4$. NON-ISOTROPIC ANTENNAS, 3 JAMMERS.

\begin{tabular}{|c|cc|r|r|r|r|}
\hline \multirow{2}{*}{$\begin{array}{c}\text { SP } \\
\text { Method }\end{array}$} & \multicolumn{5}{|c|}{ Parameter estimation via Monte Carlo simulation } \\
\cline { 2 - 7 } & \multicolumn{2}{|c|}{$c \cdot \delta \tau_{0}$} & \multicolumn{2}{|c|}{$\delta \psi_{0}$} & \multicolumn{1}{c|}{ CNIR } & \multicolumn{1}{c|}{ ISR } \\
\cline { 2 - 7 } & mean & std & mean & \multicolumn{1}{c|}{ std } & mean & mean \\
\hline PA & $2.99 \mathrm{~m}$ & $0.43 \mathrm{~m}$ & $97.8^{\circ}$ & $41.94^{\circ}$ & 29.9 & 39.7 \\
DB & $2.96 \mathrm{~m}$ & $0.32 \mathrm{~m}$ & $0.0^{\circ}$ & $0.02^{\circ}$ & 44.7 & -85.3 \\
SC & $2.98 \mathrm{~m}$ & $0.45 \mathrm{~m}$ & $0.1^{\circ}$ & $2.1^{\circ}$ & 37.2 & 29.8 \\
PI & $3.02 \mathrm{~m}$ & $0.23 \mathrm{~m}$ & $-88.5^{\circ}$ & $45.80^{\circ}$ & 50.4 & -22.5 \\
MVDR & $3.00 \mathrm{~m}$ & $0.14 \mathrm{~m}$ & $4.4^{\circ}$ & $6.12^{\circ}$ & 50.8 & -20.3 \\
LMS & $3.03 \mathrm{~m}$ & $0.14 \mathrm{~m}$ & $0.1^{\circ}$ & $0.01^{\circ}$ & 51.0 & -20.8 \\
\hline
\end{tabular}

Results suggest that there is not as much influence of selected SP method for interference/jamming mitigation compared with the effect of antenna array plus RF front-end transfer function. It is clearly seen that results in terms of phase and group delays are almost the same as in previous simulation. This indicates the need of proper compensation techniques that produce an equalization of the transfer function at DOA of signal of interest. Particularly, for PA and SC methods the ISR is so high that signal could hardly be recovered in a real situation. Phase and group delays can be obtained only because CRPA can be precomputed in an offline simulation and updated with the particular set of weight vector coefficients. It also can be noted that for a blind method as PI is, such biases are always uncontrolled because of not existing a particular restriction for some known characteristic of the received signal of interest.

\section{CONCLUding REMARKS}

Determination of the travel time of satellite signals is the main objective for GNSS receivers. For precise applications it has to be determined with $0.1 \sim 1 n s$ accuracy, meaning centimeter or sub-centiemeter accuracy for the solution of position, and uncertainties of CRPA must be solved to achieve range level of precision. The CRPA influence can be on the order of $10 \mathrm{~ns}$ (meters level) as verified in this work with a 
simulated model. The problem of obtaining an interferencefree signal subspace in a harmful scenario can be solved in many ways, but the secondary effects of these methods can be detrimental to the main objective of time of travel determination. Therefore, the influence of antennas and RF front-end on group and phase delays cannot be neglected.

Many existing compensation methods for minimizing antenna differences are based in a narrowband assumption, and in that case no restrictions are set to the total antenna array frequency response at DOA/s of interest. Then, they are not well behaved for GNSS precision applications. Therefore, a wideband model needs to be applied in order to estimate such phase and group delay biases. For example, bias compensation can be performed by applying precomputed code phase and carrier phase bias corrections to the GNSS receiver tracking estimates. These corrections can be defined as the code phase and carrier phase bias errors from their nominal non-biased values and can be determined through a calibration procedure typically including some combination of software simulation, anechoic chamber characterization, and outdoor live signal testing. Moreover, it could be of much interest to develop wideband methods for antenna arrays which do not need a complete and precise knowledge of all CRPA information, to get a robust array processing strategy.

\section{ACKNOWLEDGMENT}

This work was financed by CONICET, ANPCyT PICT 2014-1232 and UNLP Proyecto I-209.

\section{REFERENCES}

[1] E. Kaplan and C. J. Hegarty, Understanding GPS. Principles and applications, 2nd ed. Boston - London: Artech House, 2006.

[2] H. L. Van Trees, Optimum Array Processing - Detection, Estimation and Modulation Theory. Wiley, 2002.

[3] C. Fernandez-Prades, J. Arribas, and P. Closas, "Robust GNSS Receivers by Array Signal Processing: Theory and Implementation," Proceedings of the IEEE, vol. 104, no. 6, pp. 1207-1220, 2016. [Online]. Available: http://ieeexplore.ieee.org/lpdocs/epic03/wrapper.htm?arnumber=7444116

[4] D. S. De Lorenzo, S. C. Lo, P. K. Enge, and J. Rife, "Calibrating adaptive antenna arrays for high-integrity GPS," GPS Solutions, vol. 16, no. 2, pp. 221-230, 2012.

[5] E. A. Marranghelli, G. R. L. La Valle, and P. A. Roncagliolo, "Mutual coupling and electromagnetic diffraction influence on GPS microstrip antenna arrays," in 2015 16th Workshop on Information Processing and Control, RPIC 2015, 2016

[6] J. L. Volakis, A. J. O'Brien, and C. C. Chen, "Small and Adaptive Antennas and Arrays for GNSS Applications," Proceedings of the IEEE, vol. 104, no. 6, pp. 1221-1232, 2016.

[7] S. Irteza, E. Schäfer, M. Sgammini, R. Stephan, and M. Hein, "FourElement Compact Planar Antenna Array for Robust Satellite Navigation Systems," in EuCAP, 2013, pp. 19-23.

[8] C. Craeye and D. González-Ovejero, "A review on array mutual coupling analysis," Radio Science, vol. 46, no. 2, pp. 1-25, 2011.

[9] D. Kelley and W. Stutzman, "Array antenna pattern modeling methods that include mutual coupling effects," IEEE Transactions on Antennas and Propagation, vol. 41, no. 12, pp. 1625-1632, 1993.

[10] T. Sarkar, M. Wicks, and R. Bonneau, Smart Antennas. New Jersey: Wiley, 2005.

[11] S. Henault and Y. Antar, "Unifying the Theory of Mutual Coupling Compensation in Antenna Arrays," IEEE Antennas and Propagation Magazine, vol. 57, no. 2, pp. 104-122, 2015.

[12] S. K. Tiong, B. Salem, S. P. Koh, K. P. Sankar, and S. Darzi, "Minimum variance distortionless response (MVDR) beamformer with enhanced nulling level control via dynamic mutated artificial immune system," The Scientific World Journal, 2014.
[13] H. Zhao, B. Lian, and J. Feng, "Adaptive beamforming and phase bias compensation for GNSS receiver," Journal of Systems Engineering and Electronics, vol. 26, no. 1, pp. 10-18, 2015.

[14] C. L. Chang, "Self-tuning synthesis filter against mutual coupling and interferences for GNSS and its implementation on embedded board," Eurasip Journal on Advances in Signal Processing, vol. 2010, 2010.

[15] X. Chen, C. G. Parini, B. Collins, Y. Yao, and M. Ur Rehman, Antennas for Global Navigation Satellite Systems, 2012. [Online]. Available: http://doi.wiley.com/10.1002/9781119969518

[16] P. Anantharamu, D. Borio, and G. Lachapelle, "Self-Contained Antenna Array Calibration Using GNSS Signals," NAVIGATION J. Inst. Navig., vol. 59, pp. 209-220, 2012.

[17] I. Progri, Geolocation of RF signals: Principles and simulations, 2011.

[18] E. Marranghelli, G. La Valle, and P. Roncagliolo, "Método de compensación de acoplamiento mutuo y difracción electromagnética en arreglos de antenas de microtira para GNSS," in 2016 IEEE Biennial Congress of Argentina, ARGENCON 2016, 2016.

[19] S. Daneshmand, N. Sokhandan, M. Zaeri-Amirani, and G. Lachapelle, "Precise Calibration of a GNSS Antenna Array for Adaptive Beamforming Applications," Sensors, pp. 9669-9691, 2014. [Online]. Available: www.mdpi.com/journal/sensors

[20] U. Kim and D. D. Lorenzo, "Phase effects analysis of patch antenna CRPAs for JPALS," ION GNSS 17th International Technical Meeting of the Satellite Division, no. September, pp. 1531-1538, 2004.

[21] U. S. Kim, "Analysis of Carrier Phase and Group Delay Biases Introduced by CRPA Hardware," Proceedings of the 18th International Technical Meeting of the Satellite Division of The Institute of Navigation, pp. 635-642, 2005.

[22] A. J. O'Brien and I. J. Gupta, "An optimal adaptive filtering algorithm with zero antenna-induced bias for GNSS antenna arrays," Navigation, Journal of the Institute of Navigation, vol. 57, no. 2, pp. 87-100, 2010.

[23] I. J. Gupta, I. M. Weiss, and A. W. Morrison, "Desired Features of Adaptive Antenna Arrays for GNSS Receivers," Proceedings of the IEEE, vol. 104, no. 6, pp. 1195-1206, 2016.

[24] A. Konovaltsev, M. Cuntz, L. A. Greda, M. V. T. Heckler, and M. Meurer, "Antenna and RF front end calibration in a GNSS array receiver," 2010 IEEE International Microwave Workshop Series on "RF Front-ends for Software Defined and Cognitive Radio Solutions", IMWS 2010 - Proceedings, pp. 103-106, 2010.

[25] S. Backén, D. Akos, and M. Nordenvaad, "Post-processing dynamic GNSS antenna array calibration and deterministic beamforming," ION GNSS, 2008.

[26] S. Daneshmand, A. Jahromi, A. Broumandan, and G. Lachapelle, "GNSS Space-Time Interference Mitigation and Attitude Determination in the Presence of Interference Signals," Sensors, vol. 15, no. 6, pp. 12 180-12 204, 2015. [Online]. Available: http://www.mdpi.com/1424$8220 / 15 / 6 / 12180 /$

[27] A. Konovaltsev, S. Caizzone, M. Cuntz, and M. Meurer, "Autonomous Spoofing Detection and Mitigation with a Miniaturized Adaptive Antenna Array," Proceedings of the 27th International Technical Meeting of the Satellite Division of The Institute of Navigation, ION GNSS+ 2014, Tampa, Florida, September 8-12, 2014, pp. 2937-2948, 2014.

[28] M. Cuntz, A. Konovaltsev, and M. Meurer, "Concepts, Development, and Validation of Multiantenna GNSS Receivers for Resilient Navigation," Proceedings of the IEEE, 2016.

[29] M. Sgammini, F. Antreich, L. Kurz, M. Meurer, and T. G. Noll, "Blind Adaptive Beamformer Based on Orthogonal Projections for GNSS," Proceedings of the 25th International Technical Meeting of The Satellite Division of the Institute of Navigation, no. 1, pp. 926-935, 2012.

[30] C. A. Balanis, Antenna Theory, Analysis and Design. Wiley, 1982.

[31] Rogers, "RT/duroid 6002 High Frequency Laminates," 2011.

[32] Weiland, "A discretization method for the solution of Maxwell's equations for six component fields: Electronics and Communication," Int. J. Electron. Comun. (AEU), vol. 31, no. 3, pp. 116-120, 1977. 